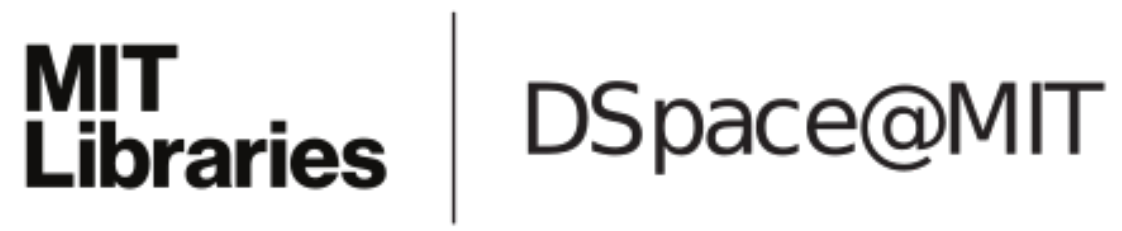

\author{
MIT Open Access Articles
}

Mixing in a liquid metal electrode

The MIT Faculty has made this article openly available. Please share how this access benefits you. Your story matters.

Citation: Kelley, Douglas H., and Donald R. Sadoway. "Mixing in a Liquid Metal Electrode." Physics of Fluids 26, no. 5 (May 2014): 057102. ( 2014 AIP Publishing LLC

As Published: http://dx.doi.org/10.1063/1.4875815

Publisher: American Institute of Physics (AIP)

Persistent URL: http://hdl.handle.net/1721.1/92268

Version: Final published version: final published article, as it appeared in a journal, conference proceedings, or other formally published context

Terms of Use: Article is made available in accordance with the publisher's policy and may be subject to US copyright law. Please refer to the publisher's site for terms of use. 


\section{AIP $\mid$ Physics of Fluids}

\section{Mixing in a liquid metal electrode}

Douglas H. Kelley and Donald R. Sadoway

Citation: Physics of Fluids (1994-present) 26, 057102 (2014); doi: 10.1063/1.4875815

View online: http://dx.doi.org/10.1063/1.4875815

View Table of Contents: http://scitation.aip.org/content/aip/journal/pof2/26/5?ver=pdfcov

Published by the AIP Publishing

\section{Articles you may be interested in}

Electrochemical interface between an ionic liquid and a model metallic electrode

J. Chem. Phys. 126, 084704 (2007); 10.1063/1.2464084

Measurements of the magnetic field induced by a turbulent flow of liquid metala)

Phys. Plasmas 13, 055901 (2006); 10.1063/1.2173614

High-Reynolds-number simulation of turbulent mixing

Phys. Fluids 17, 081703 (2005); 10.1063/1.2001690

Compressibility effects on turbulence structures of axisymmetric mixing layers

Phys. Fluids 15, 1755 (2003); 10.1063/1.1570829

A study on a lobed jet mixing flow by using stereoscopic particle image velocimetry technique

Phys. Fluids 13, 3425 (2001); 10.1063/1.1409537

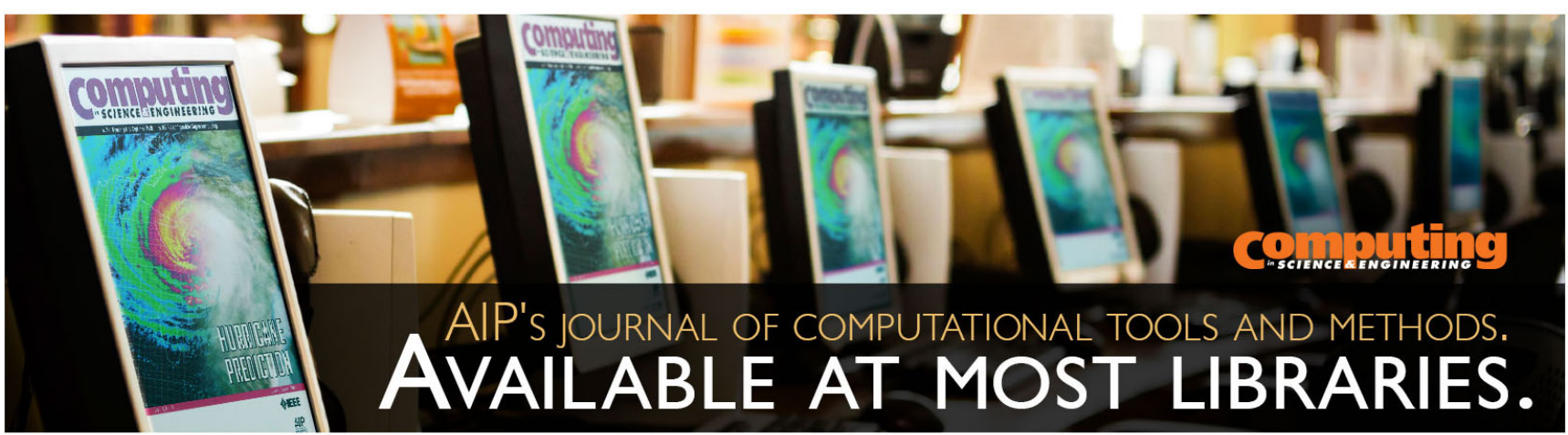




\title{
Mixing in a liquid metal electrode
}

\author{
Douglas H. Kelley ${ }^{1, a)}$ and Donald R. Sadoway ${ }^{2}$ \\ ${ }^{1}$ Department of Mechanical Engineering, University of Rochester, Rochester, \\ New York 14627-0132, USA \\ ${ }^{2}$ Department of Materials Science and Engineering, Massachusetts Institute of Technology, \\ Cambridge, Massachusetts 02139, USA
}

(Received 7 January 2014; accepted 29 April 2014; published online 20 May 2014)

Fluid mixing has first-order importance for many engineering problems in mass transport, including design and optimization of liquid-phase energy storage devices. Liquid metal batteries are currently being commercialized as a promising and economically viable technology for large-scale energy storage on worldwide electrical grids. But because these batteries are entirely liquid, fluid flow and instabilities may affect battery robustness and performance. Here we present estimates of flow magnitude and ultrasound measurements of the flow in a realistic liquid metal electrode. We find that flow does substantially affect mass transport by altering the electrode mixing time. Above a critical electrical current density, the convective flow organizes and gains speed, which promotes transport and would yield improved battery efficiency. (c) 2014 AIP Publishing LLC. [http://dx.doi.org/10.1063/1.4875815]

\section{INTRODUCTION}

The need for sustainable energy is bringing new emphasis to industrial applications of the physics of fluids. In technologies as diverse as wind power, ${ }^{1}$ tidal generation, ${ }^{2}$ and energy harvesting, ${ }^{3}$ optimizing performance depends on a careful and thorough understanding of the underlying flow phenomena. Nor is power production the only application; the operation of energy storage devices like fuel cells ${ }^{4-6}$ and flow batteries, ${ }^{7,8}$ for example, is fundamentally linked to fluid flow.

In this paper, we focus on fluid dynamical effects in a new energy storage device, the liquid metal battery. Recently proposed and currently the topic of intense research ${ }^{9,10}$ and development, ${ }^{11}$ liquid metal batteries are being designed for storing large amounts of energy on the world's electrical grids. Installing substantial grid-scale storage would ease grid management and reduce costly load variations. Moreover, adding storage would allow for the widespread utilization of sustainable energy sources, such as wind, solar, and tidal power, which are inherently intermittent. Liquid metal batteries might soon allow grid operators to store away solar power for a rainy day, or wind power for a quiescent one.

But liquid metal batteries differ from conventional batteries because the electrolyte and both electrodes operate in the liquid state, bringing flow physics to bear on design and implementation. Moreover, a number of processes in the batteries can drive flow. First, keeping the batteries liquid requires maintaining elevated temperatures and unavoidably introduces thermal gradients and therefore thermal convection. Second, these batteries accommodate large electrical currents which in turn cause electromagnetic (Lorentz) forces. Additional thermal gradients can arise locally from ohmic and entropic heating. Finally, when new materials are produced whose density differs from their surroundings, natural (compositional) convection may occur as well. Given the complexity of the relevant phase diagrams, however, we leave consideration of compositional convection to future work. Once initiated, flow in liquid metal batteries can affect their performance and robustness in a variety of ways.

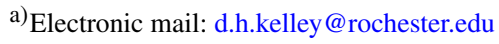


Here we study flow in liquid metal batteries and consider its effects on their performance and robustness. Previous studies of thermal convection in liquid metals ${ }^{12-17}$ have established a robust theoretical understanding but have usually focused on geophysical and astrophysical applications, not on energy technology. The monograph by Bojarevičs ${ }^{18}$ summarizes previous studies of flow driven by electrical currents (sometimes called electrically-induced vortex flow), which are fewer. ${ }^{19-21}$ Rarely have both convection and electrically-induced vortex flow been considered simultaneously. Using the equations of motion, we make order-of-magnitude estimates of the relative impact of each of the forcing mechanisms mentioned above. Using ultrasound velocimetry measurements in a liquid metal electrode, we quantify and characterize the flows driven by thermal convection in the presence of electrical currents. Ultrasound is regularly used in liquid metal, ${ }^{22-26}$ but to our knowledge, it has not been used previously in an electrode with currents applied. We relate flow to battery performance by considering mass transport, and specifically by measuring the characteristic mixing time of the flow as a function of current density. We find that above a critical current density, the convective flow organizes and gains speed, leading to smaller mixing times and improved mass transport. Our results predict that the performance of liquid metal batteries improves substantially when current density is maintained above this critical value, roughly $50 \mathrm{~mA} / \mathrm{cm}^{2}$, which is well below the current densities typical in operation. We conclude by discussing methods to leverage this prediction.

\section{OVERVIEW OF LIQUID METAL BATTERIES}

A typical liquid metal battery is shown in Fig. 1. The design and operation of these systems have been reviewed previously from the perspectives of materials science and electrochemistry; ${ }^{10}$ here we focus on the role of flow physics in battery operation. The negative electrode is composed of a metal with low electronegativity (electron donor, here sodium), typically having low mass density. The positive electrode is composed of a metal with high electronegativity (electron acceptor, here the eutectic alloy of lead and bismuth, referred to below as ePbBi), typically having high mass density. Owing to the donor/acceptor pairing, alloying of the metals is energetically favorable. To control alloying electrochemically, the electrodes are separated by a molten salt electrolyte that allows the passage of ions but not neutral species. The reaction can proceed only if each itinerant metal ion is accompanied by its dissociated electron(s) which flow through an external circuit, providing electrical power. Thus alloying the metals discharges the battery, and the process can be reversed to charge the battery by supplying external electrical power.

Because we choose electrolyte materials that have mass density intermediate between the negative and positive electrodes, and because electrolytes are axiomatically insoluble in metals, stable stratification naturally segregates the three layers of a liquid metal battery. Density stratification obviates the need for solid separators (which are delicate and can limit battery lifetime) or paste electrolytes (which transport ions much more slowly than molten salts). The electrodes themselves are consumed and regenerated during cycling, eliminating the structural degradation due to swelling and contraction that limits the lifetime of many batteries, particularly those with Li-ion chemistry. ${ }^{27,28}$
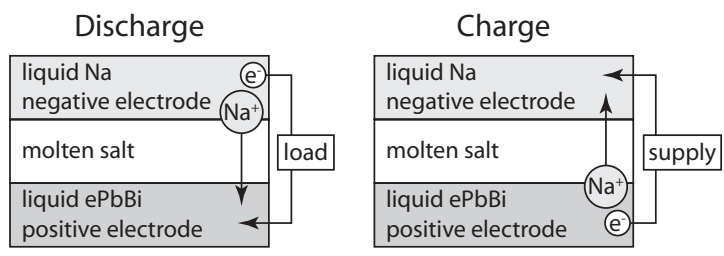

FIG. 1. Operation of a liquid metal battery. During discharge, one metal $(\mathrm{Na})$ is allowed to alloy with another (ePbBi) by transferring ions through a molten salt electrolyte and transferring compensating electrons through an external circuit, yielding electrical power. During charge, an external energy supply forces current through the cell, thereby reversing the process and regenerating the negative electrode. Because both metals and the electrolyte operate in the liquid phase, fluid processes are inherently present and may affect performance. 
But without solid surfaces or separators, surface deformations are possible, conceivably allowing contact between the electrodes, which would cause rapid discharge. Liquid metal battery designs must prevent such discharge. One fluid dynamical mechanism for surface deformation is the Tayler instability, ${ }^{29,30}$ a magnetohydrodynamic phenomenon that occurs when the azimuthal magnetic field $B_{\phi}$ satisfies the condition

$$
\frac{\partial}{\partial r}\left(r B_{\phi}^{2}\right)<0
$$

where $r$ is the cylindrical radial coordinate. The Tayler instability has been observed numerically ${ }^{31}$ and experimentally, ${ }^{32}$ and designs have been proposed ${ }^{33}$ to altogether avoid the Tayler instability in liquid metal batteries. However, its onset occurs at current densities near $30 \mathrm{~A} / \mathrm{cm}^{2}$, whereas current densities in liquid metal batteries are typically $0.3 \mathrm{~A} / \mathrm{cm}^{2}$ or less. Thus liquid metal battery technology has not yet advanced to operating regimes where we expect the Tayler instability to play a role, and we leave further study of the Tayler instability to future work.

In this paper, we will focus on flow physics relating to mass transport in liquid metal batteries. Because the electrochemical reactions through which energy is stored and released take place at the interfaces between the three battery layers, flow governs reaction rates by transporting materials to and from those interfaces. During discharge, ions arrive at the top surface of the positive electrode, where electron transfer reactions reduce them to neutral metal, which subsequently must be thoroughly mixed throughout the electrode if the full capacity of the battery is to be utilized. Likewise, during charge itinerant ions form at the electrode surface by electro-oxidation, and that reaction can be sustained only by concurrent movement of dissolved solute metal to the top surface from the bulk of the electrode. Though mass diffusion can eventually produce this mixing, achieving practical rates of discharge and charge requires faster mixing via convective transport. Battery rate capability also depends on the inherent rates of charge transfer at the electrode-electrolyte interface, but these have been determined to be very fast in systems comprising liquid metals and molten salts, especially at elevated temperatures. ${ }^{34}$ Indeed, mass transport associated with both alloying (ingress of metal solute) and dealloying (egress of metal solute) dominates under a variety of conditions.

Mass transport also affects battery resilience. When two metals are alloyed, their phase diagram often includes phases whose electrochemical properties vary widely, and some of those phases may not be desirable for battery operation. Fast mixing ensures that the composition of the alloy is homogeneous, preventing undesired phases from arising prematurely in isolated regions.

Our colleagues have performed fundamental electrochemical analyses of a variety of liquid metals ${ }^{35-37}$ and have constructed liquid metal batteries using many of those metals, including magnesium and antimony, ${ }^{9}$ lithium and a lead-antimony alloy, ${ }^{10}$ and calcium and bismuth. ${ }^{38}$ In this work, we focus on liquid metal batteries using sodium and ePbBi, as shown in Fig. 1. The advantage of this chemistry is its low operating temperature: sodium is molten above $97^{\circ} \mathrm{C}$, and eutectic $44 \%$ lead $56 \%$ bismuth is molten above $125^{\circ} \mathrm{C}$. Combining those electrodes with an electrolyte composed of a triple-eutectic combination of sodium iodide (10\%), sodium hydroxide (38\%), and sodium amide (52\%) which is molten above $127^{\circ} \mathrm{C}$, we can operate liquid metal batteries at $150^{\circ} \mathrm{C}$ with negligible chance of local freezing. For comparison, the lithium and lead-antimony combination is typically operated at $550^{\circ} \mathrm{C}$. In an industrial setting, lower temperatures have the potential to reduce operating costs substantially and extend service lifetime. Low-temperature chemistries cannot yet match the performance of high-temperature ones, but we have successfully discharged and charged a sodium-ePbBi battery repeatedly, and low-temperature performance is a topic of ongoing research among our colleagues. In the laboratory, low temperatures make experiments much more practical, in particular allowing us to use common ultrasound velocimetry probes which cannot function at $550^{\circ} \mathrm{C}$. During discharge of a liquid metal battery made from sodium and $\mathrm{PbBi}$, sodium is oxidized at the interface between the electrolyte and negative electrode. The resulting sodium ions pass through the electrolyte and are reduced at the ePbBi positive electrode, forming an alloy of sodium and $\mathrm{ePbBi}$. We measure the associated electrochemical potential to be $0.83 \mathrm{~V}$. During charge, the process is reversed.

Liquid metal batteries are attractive for grid-scale storage in part because of their high rate capability. ${ }^{10}$ Molten salt electrolytes offer ion conductivities orders of magnitude greater than 
aqueous electrolytes common in current battery technologies. Electrochemical measurements suggest that the rate-limiting process for charge and discharge of liquid metal batteries is mass transport in the positive electrode. For example, one recent study considered calcium-bismuth electrodes as a potential liquid metal battery chemistry. The study used a three-electrode electrochemical setup to measure not just the potential between the positive and negative electrodes, but the potential of each electrode with respect to a stable reference. The study found that only $10 \%$ of the voltage drop was attributable to ohmic resistance, but 59\% came from mass transport limitations. ${ }^{38}$ That is, the majority of the losses arose because mass transport supplied the interface with unreacted material and removed reacted material too slowly. For that reason and because of the complex phase diagram that governs alloy formation, we have focused our study on mixing of the positive electrode. The remainder of this paper will consider the positive electrode only.

\section{EQUATIONS OF MOTION AND DIMENSIONLESS PARAMETERS}

The molten metal in the positive electrode, which is our focus henceforth, follows known equations of motion. It can accurately be modeled as incompressible $(\nabla \cdot \boldsymbol{u}=0$, where $\boldsymbol{u}$ is the velocity), and its momentum must be conserved:

$$
\frac{\partial \boldsymbol{u}}{\partial t}+(\nabla \cdot \boldsymbol{u})=v \nabla^{2} \boldsymbol{u}-\frac{1}{\rho} \nabla p+\boldsymbol{a}_{b}+\boldsymbol{a}_{L} .
$$

Here $t$ is time, $v=2.79 \times 10^{-7} \mathrm{~m}^{2} / \mathrm{s}$ is the kinematic viscosity, $\rho=10.54 \mathrm{~g} / \mathrm{cm}^{3}$ is the density, $p$ is the pressure, and the thermal buoyant acceleration $\boldsymbol{a}_{b}$ and Lorentz acceleration $\boldsymbol{a}_{L}$ are given by

$$
\begin{aligned}
\boldsymbol{a}_{b} & =\alpha \Delta T g, \text { and } \\
\boldsymbol{a}_{L} & =\frac{1}{\mu_{0} \rho}(\nabla \times \boldsymbol{B}) \times \boldsymbol{B},
\end{aligned}
$$

respectively, where $\alpha=4.24 \times 10^{-5} \mathrm{~K}^{-1}$ is the coefficient of linear expansion, $\Delta T$ is the temperature differential, $g=9.8 \mathrm{~m} / \mathrm{s}^{2}$ is the gravitational acceleration, $\mu_{0}$ is the magnetic constant, and $\boldsymbol{B}$ is the magnetic field. All material properties are evaluated at $150{ }^{\circ} \mathrm{C}$ using the expressions published by the Nuclear Energy Agency. ${ }^{39}$ Though solving this equation of motion analytically or numerically is outside the scope of this work, it is possible to estimate the order of magnitude of both $\boldsymbol{a}_{b}$ and $\boldsymbol{a}_{L}$. If the temperature differential is $\Delta T=10^{\circ} \mathrm{C}$ (consistent with our observations in the experiments described below and also consistent with previous experiments by our collaborators), then $\left|\boldsymbol{a}_{b}\right| \sim 1 \mathrm{~mm} / \mathrm{s}^{2}$.

Estimating the Lorentz acceleration is more complicated. If we approximate the current density as purely axial and uniform throughout the area of the negative current collector, and further ignore fringing fields, we can estimate the magnetic field with Ampère's law,

$$
2 \pi R|\boldsymbol{B}|=\mu_{0} \pi R^{2} J
$$

where $R$ is the radius of the positive electrode and $J=|\boldsymbol{J}|$ is the magnitude of the current density. Solving Eq. (3) for $|\boldsymbol{B}|$ and asserting that the spatial variation in $|\boldsymbol{B}|$ has some characteristic length scale $L_{B}$, we can write the order of magnitude of the Lorentz acceleration as

$$
\boldsymbol{a}_{L} \sim \frac{\mu_{0} R^{2} J^{2}}{4 \rho L_{B}} .
$$

Choosing $J \sim 275 \mathrm{~mA} / \mathrm{cm}^{2}$ (as is commonly done for prototypes) and hypothesizing that $\boldsymbol{B}$ varies over spatial scales of order $0.1 \mathrm{~mm}$, we find that $\left|\boldsymbol{a}_{L}\right| \sim 1 \mathrm{~mm} / \mathrm{s}^{2}$ as well. Such small-scale variations in the magnetic field are likely, particularly near the negative current collector, which has sharp corners of the sort that concentrate fields. That said, our estimates for $\left|\boldsymbol{a}_{L}\right|$ and $\left|\boldsymbol{a}_{b}\right|$ come from order-of-magnitude empirical observations and should not be attributed with high accuracy. The current density in liquid metal batteries is not uniform, nor does uniform current cause flow. ${ }^{18}$ Our estimates do not provide scaling laws capable of predicting electrode dynamics over a wide range of operating parameters. But they do suggest that at the particular parameters common for prototypes, 
neither the buoyant acceleration nor the Lorentz acceleration could accurately be ignored in future models.

We can also estimate dimensionless quantities that characterize the system. The Rayleigh number is the normalized buoyancy, given by

$$
R a=\frac{g \alpha \Delta T L^{3}}{\nu \kappa} \sim 10^{4},
$$

where $L=16 \mathrm{~mm}$ is the electrode thickness and $\kappa=6.15 \times 10^{-6} \mathrm{~m}^{2} / \mathrm{s}$ is the thermal diffusivity of ePbBi. ${ }^{39}$ Because $R a \gg 1$, we would expect unsteady convective flow in this system. ${ }^{40}$ The Prandtl number is the ratio of momentum diffusion to thermal diffusion, given by $\operatorname{Pr}=\nu / \kappa$; for $\mathrm{ePbBi}$, $\operatorname{Pr}=0.045$. In the case of zero current $\left(\left|\boldsymbol{L}_{b}\right|=0\right)$, thermal convection can be parameterized in terms of $R a$ and $P r$ alone. In that case, scaling arguments ${ }^{16}$ predict that our system operates in a regime where both thermal dissipation and momentum dissipation occur primarily in the boundary layers (not in the bulk), and the kinetic boundary layer is thicker than the thermal boundary layer. When current is applied, an additional dimensionless parameter is required. In particular, we would expect a Hartmann boundary layer to arise in the presence of current, and the associated parameter is the Hartmann number

$$
H a=|\boldsymbol{B}| L \sqrt{\frac{\sigma}{\mu_{0}}}=\frac{1}{2} R J L \sqrt{\sigma \mu_{0}},
$$

where $\sigma=9.26 \times 10^{5} \mathrm{~S} / \mathrm{m}$ is the electrical conductivity of $\mathrm{ePbBi}$ at $150^{\circ} \mathrm{C}^{39}$ and we have used Eq. (3). In the experiments described below, $0 \leq H a \leq 1.46$. Axial currents in convecting fluids with cylindrical geometry typically smooth internal temperature variations while raising the temperature along the central axis. ${ }^{18}$ Our practical goal in this work is to control mass transport by fluid flow, and the appropriate dimensionless measure is the Reynolds number $R e=<u^{2}>{ }^{1 / 2} L / \nu$. In the experiments described below, $50 \leq R e \leq 200$. In future work, we intend to develop scaling arguments to predict the effect of applied current on electrode dynamics and express $R e$ as a function of $R a, P r$, and $H a$.

\section{METHODS}

Our experimental setup is sketched in Fig. 2. A positive electrode composed of ePbBi is contained in a cylindrical vessel with inner diameter $88.9 \mathrm{~mm}$. Made of 304 stainless steel, the vessel is $67 \mathrm{~mm}$ deep and nearly identical to vessels our group uses for electrochemical testing of battery prototypes. The design might also be viable for commercial development. The vessel serves as a positive current collector, maintaining electrical contact with the (liquid) positive electrode and

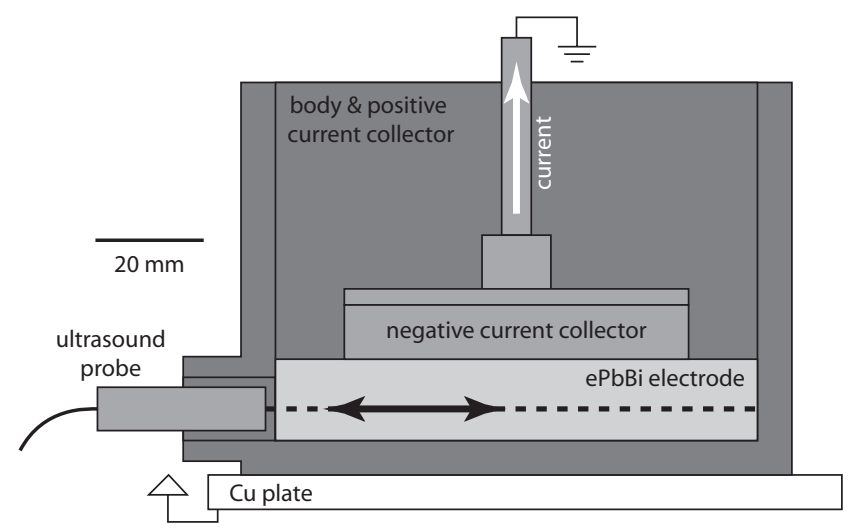

FIG. 2. Experimental setup. In this study, we focus on mixing in the positive electrode, which we study in a battery setup modified to accommodate ultrasound velocimetry. The electrode is connected to a power supply via positive and negative current collectors like those used in three-layer battery prototypes. The copper plate below the vessel ensures uniform heat flux and current density. The experiment takes place in an argon atmosphere. 
thereby allowing current flow to a load or supply. A separate negative current collector, made of nickel-iron foam, provides the second terminal. The negative current collector is cylindrical, has diameter $64 \mathrm{~mm}$, and is identical to current collectors used in prototypes. A PTFE bracket aligns its axis with the axis of the vessel. In these experiments, the axis of the negative current collector is connected to a DC power supply in current-controlled mode. From the power supply, current passes through a $49.428 \mathrm{~m} \Omega$ shunt (which allows for precise measurement of the current) and into a copper plate in contact with the vessel. The copper is $6.4 \mathrm{~mm}$ thick, and because its electrical conductivity is an order of magnitude larger than that of the stainless steel vessel or the ePbBi electrode, it ensures uniform current density. A K-type thermocouple inserted into the ePbBi layer measures its temperature. A second thermocouple in the ePbBi connects to an automated thermal system, which holds the liquid metal temperature near $150^{\circ} \mathrm{C}$. The vessel is heated from below and insulated around its sides, in a thermal arrangement similar to the one used for testing battery prototypes. The copper plate also ensures uniform heating due to its large thermal conductivity.

The vessel shown in Fig. 2 differs from most liquid metal battery prototypes, however, by including a side port for an ultrasound probe. Built on the same principles as sonar, ultrasound velocimetry has been used successfully in a variety of liquid metals. ${ }^{22-26}$ In its most common configuration, it measures one component of fluid velocity, motion toward and away from the probe, at an array of locations along the line of sight from the probe face. Thanks to the low melting point, we are able to put $\mathrm{ePbBi}$ in direct contact with an ultrasound probe, yielding good signal strength. (High-temperature ultrasound probes ${ }^{41}$ have recently been developed as well. We plan to use them with high-temperature battery chemistries in future work.) In this current work, the probe (Signal-Processing SA TR0805LTH connected to a Signal-Processing DOP 2000 velocimeter) made measurements along a horizontal chord through the center of the cylindrical vessel, $6.6 \mathrm{~mm}$ above the floor. The overall electrode thickness was $16 \mathrm{~mm}$. A key input for ultrasound velocimetry is the speed of sound in the working fluid. Our experiments take place at $150^{\circ} \mathrm{C}$; the speed of sound is $c=1780 \mathrm{~m} / \mathrm{s}$ at that temperature. ${ }^{39}$ We have also measured the sound speed using a micrometer immersed in $\mathrm{ePbBi}$, finding results similar to the published value.

Making high-quality ultrasound measurements in liquid metal requires care, however. Oxidation of the liquid metal must be minimized, as oxidation significantly reduces the signal strength. We enclose the entire apparatus sketched in Fig. 2 in an argon-filled glovebox. When preparing for experiments, we melt ePbBi in a separate crucible and skim as much oxide from the surface as possible before transferring to the test vessel, with the probe already in place. Consistent with previous work in liquid metal, we find acceptable signal levels without adding tracer particles, suggesting that trace oxide in the bulk produces sufficient echoes. ${ }^{22}$ But allowing more oxidation causes problems: the signal degrades quickly when oxide levels grow to only a small fraction of the total mass of the electrode, so we hypothesize that surface effects at the probe face are to blame. A scheme to separate the probe from the liquid metal by a thin wall, whose surface could be carefully controlled, might reduce the adverse affects of oxide. We will develop such a scheme in future work.

\section{RESULTS}

In a typical series of experiments, once we have added ePbBi to the vessel and put the negative current collector in place, we wait for the liquid metal to become quiescent before applying current. We hold the current steady for at least $300 \mathrm{~s}$, recording ultrasound measurements throughout that time. A typical measurement without current, in which the flow is driven by thermal convection, is shown in Fig. 3(a). In this and subsequent plots, the probe was oriented along a chord through the axial center of the vessel, as sketched in Fig. 2, in order to measure the radial component of the velocity. We denote distance from the probe with the radial coordinate $r$, choosing $r=0$ at the axial center of the cup, $r<0$ at locations between the center and the probe, and $r>0$ at locations beyond the center. In these coordinates, the far wall of the cup lies at $r=+44.5 \mathrm{~mm}$, and the near wall lies at $r=-44.5 \mathrm{~mm}$ (but is interrupted by the porthole directly in front of the probe). Measurements are recorded at an array of locations across the cup, spaced $220 \mu \mathrm{m}$ in the radial direction. Flow away from the probe is indicated with $u>0$, whereas $u<0$ signifies flow toward the probe. 

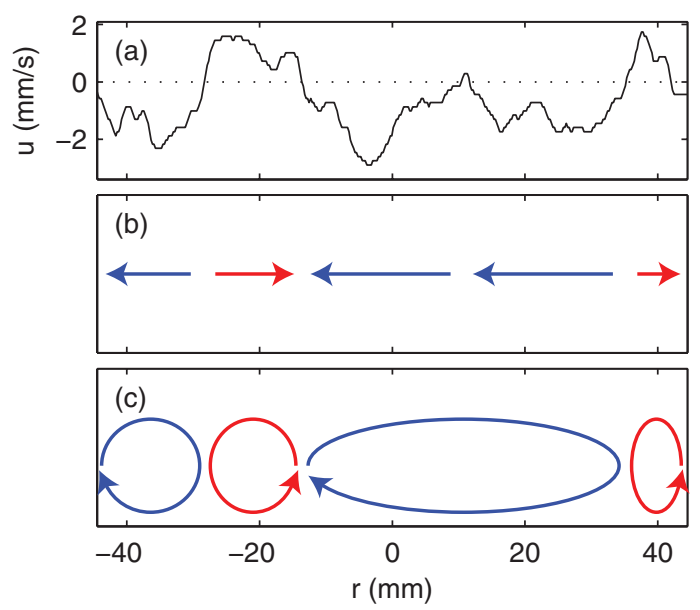

FIG. 3. An ultrasound velocity measurement and its interpretation. (a) Radial speed $u$ of thermal convection in ePbBi, varying along the radial direction $r$. (b) $u<0$ indicates flow toward the probe (at left in this coordinate system), whereas $u$ $>0$ indicates flow away from the probe. (c) A sketch of Bénard cells consistent with the measurement.

Figure 3(a) shows a single ultrasound trace, recorded without electrical current $(J=0)$. In that trace we observe radial flow away from the probe over the regions $-28 \mathrm{~mm} \leq r \leq-13.6 \mathrm{~mm}$ and $35.2 \mathrm{~mm} \leq r \leq 42 \mathrm{~mm}$. Elsewhere we observe radial flow toward the probe, except near $r=11 \mathrm{~mm}$, where there is almost no flow in the radial direction. We sketch these regions in Fig. 3(b). The velocity drops nearly to zero at the far wall $(r=44.5 \mathrm{~mm})$, as we would expect from the no-slip boundary condition. The fact that it is not identically zero could be explained by the presence of a boundary layer thinner than $220 \mu \mathrm{m}$ and therefore too small to resolve in these experiments. Because the near wall is interrupted in front of the probe to allow direct contact between the probe and liquid metal, we do not necessarily expect $u=0$ at $r=-44.5 \mathrm{~mm}$. And though we cannot be sure of the global, three-dimensional flow structure by measuring just one component of the velocity along just one line through the fluid, our observations are consistent with a slice through a set of Bénard cells like those sketched in Fig. 3(c). Our measurements do not give us access to the buoyant acceleration, but the speeds we observe are reasonable given the estimated acceleration $\left(\sim 1 \mathrm{~mm} / \mathrm{s}^{2}\right)$ and the flow variation timescales $(\sim 1 \mathrm{~s})$.

In Fig. 3, we plotted speed on the vertical axis. By indicating speed with color instead, we can plot time on the vertical axis and juxtapose a sequence of observations, producing a space-time graph (kymograph) like the one shown in Fig. 4(a). The graph spans $56 \mathrm{~s}$ of continuous measurement, and the horizontal axis remains unchanged from Fig. 3. The flow is aperiodic and disordered in both space and time, matching our expectations from the Rayleigh number (Eq. (4)) and consistent with canonical studies of thermal convection. ${ }^{40}$ The mean flow during this $56 \mathrm{~s}$ observation time is plotted in Fig. 4(b). Because the flow is unsteady, the mean is often dominated by isolated or intermittent events. The standard deviation about the mean, also plotted if Fig. 4(b), is typically similar in magnitude to the global root-mean-square speed $\left\langle u^{2}\right\rangle^{1 / 2}$, which averages over the entire $56 \mathrm{~s}$ and the entire radius range, and is one measure of the characteristic speed of the flow. Thus temporal variations are strong.

But when we introduce substantial electrical current, the flow characteristics change. Figure 5 shows flow measurements in the same electrode as Fig. 4, but with current density $J=49.9 \mathrm{~mA} / \mathrm{cm}^{2}$ passing through the electrode. (Here and throughout, we quantify the current density at the base of the electrode. For example, $49.9 \mathrm{~mA} / \mathrm{cm}^{2}$ is equivalent to $3.10 \mathrm{~A}$ distributed across the electrode base, which has diameter $88.9 \mathrm{~mm}$.) Whereas in some regions of the electrode we observe similarly disordered flow, over $10 \mathrm{~mm} \leq r \leq 44.5 \mathrm{~mm}$ the flow is much more steady than without current. Its steady character is visible in the space-time graph (Fig. 5(a)) and also indicated by the small deviation (roughly $25 \%$ of the root-mean-square speed) plotted in Fig. 5(b). Again the observed velocities are reasonable given the order-of-magnitude estimates of $\boldsymbol{a}_{b}$ and $\boldsymbol{a}_{L}$. 


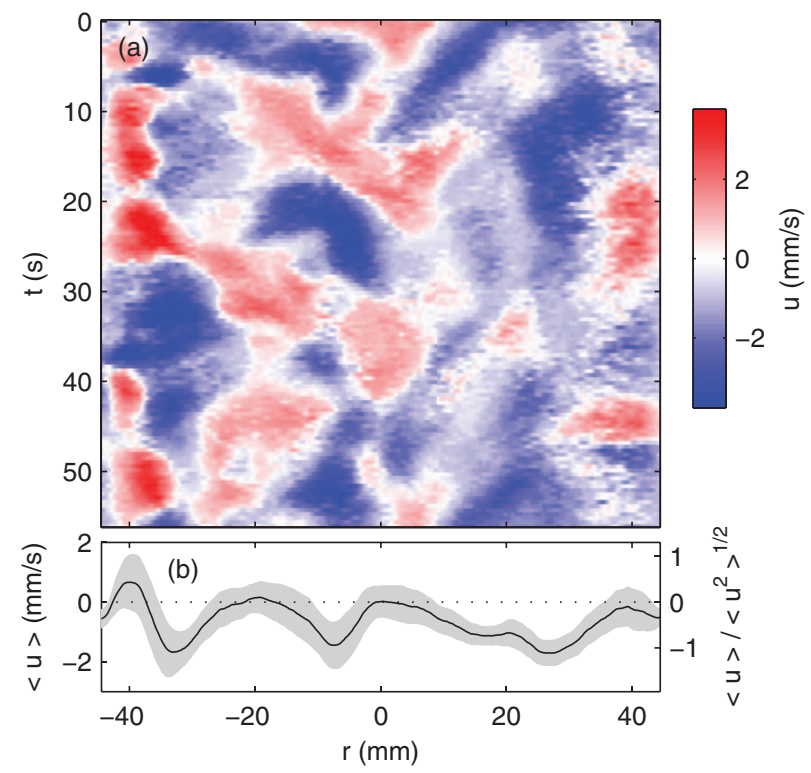

FIG. 4. Ultrasound velocity measurements of a liquid metal electrode driven by thermal convection, without electrical current. (a) Radial speed varying in both space and time, with speed indicated in color. Here $r$ is the radial coordinate and $t$ is time. (b), Mean radial speed, from the $56 \mathrm{~s}$ of measurements shown in (a). The gray region spans one standard deviation. The disorder in space and time apparent here is characteristic of thermal convection.

The changes become more evident as we increase the current density further. Figure 6 shows the situation with current density $J=125 \mathrm{~mA} / \mathrm{cm}^{2}$. Here the entire flow has become more ordered and nearly steady, apparently comprising one or two large Bénard cells. (The dip near $r=12 \mathrm{~mm}$ might indicate a nearby boundary between cells or some deformation in a single cell.) The deviation is now consistently small, often below $25 \%$. The flow is also faster-note the

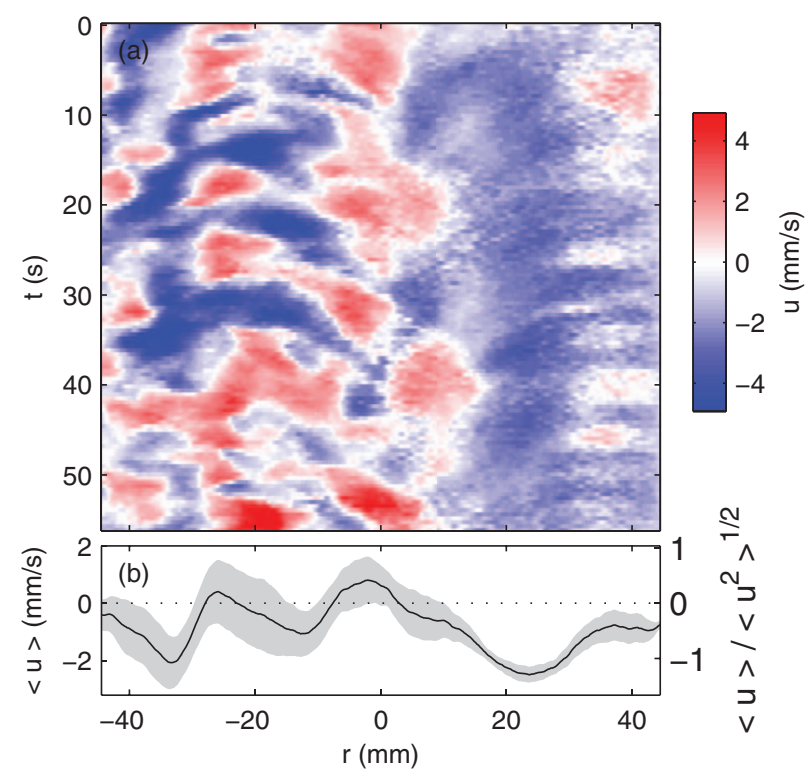

FIG. 5. Ultrasound velocity measurements of a liquid metal electrode driven by thermal convection and electrical current density $J=49.9 \mathrm{~mA} / \mathrm{cm}^{2}(H a=0.19)$. (a) Radial speed of thermal convection in ePbBi, varying in both space and time, with speed indicated in color. (b) Mean radial speed, with gray region spanning one standard deviation. At $49.9 \mathrm{~mA} / \mathrm{cm}^{2}$, the flow is disordered in some regions but ordered in others. 


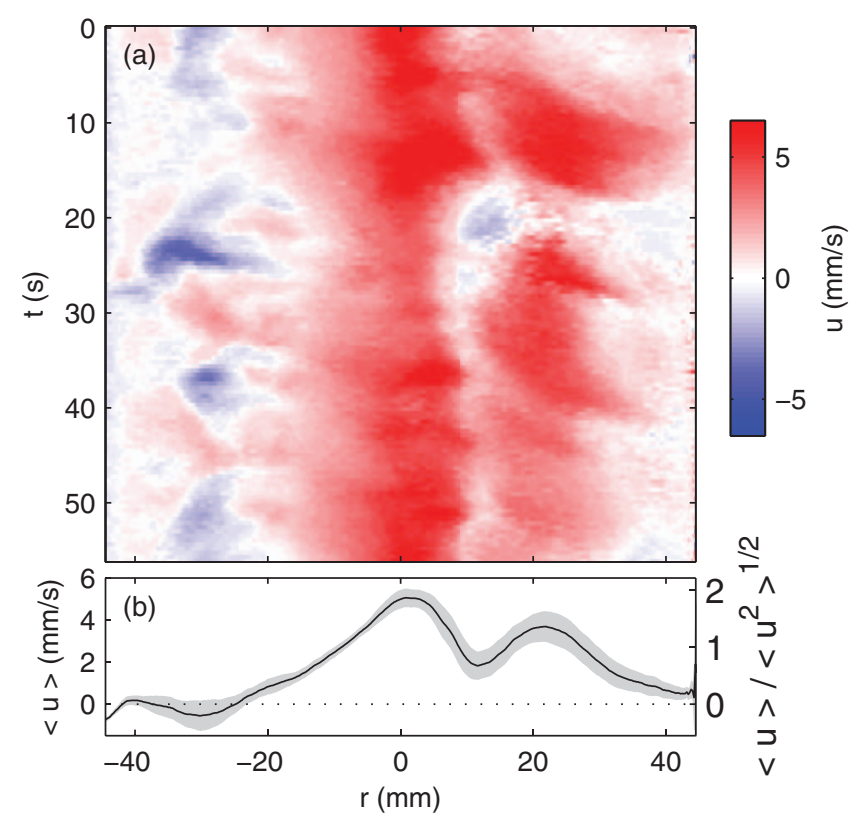

FIG. 6. Ultrasound velocity measurements of a liquid metal electrode driven by thermal convection and electrical current density $J=125 \mathrm{~mA} / \mathrm{cm}^{2}(\mathrm{Ha}=0.49)$. (a) Radial speed of thermal convection in $\mathrm{PbBi}$, varying in both space and time, with speed indicated in color. (b) Mean radial speed, with gray region spanning one standard deviation. At $125 \mathrm{~mA} / \mathrm{cm}^{2}$, the flow is largely ordered and steady.

difference in velocity scale between Figs. 5 and 6. These characteristics are consistent with previous studies of magnetoconvection. ${ }^{42-45}$ In the presence of a horizontal magnetic field, Lorentz accelerations guide Bénard cells to align their axes with field lines. Once aligned, the cells display increased speed and reduced spatial variability. The axial current in liquid metal batteries induces azimuthal, horizontal magnetic field lines. Accordingly, we would expect larger speeds and reduced temporal variation as the field increases, consistent with the results shown in Figs. 5 and 6.

A large region in which we observed flow toward the probe with current density $49.9 \mathrm{~mA} / \mathrm{cm}^{2}$ (Fig. 5) shows flow away from the probe with current density $125 \mathrm{~mA} / \mathrm{cm}^{2}$ (Fig. 6). Reorientations, reversals, and cessations are common in convection in a cylinder, ${ }^{46-48}$ and we attribute this change to such an event.

When we increase the current density still more, new phenomena appear. Figure 7 shows flow measurements in the same electrode, with $J=350 \mathrm{~mA} / \mathrm{cm}^{2}$. Though the speed remains high (near $2\left\langle u^{2}\right\rangle^{1 / 2}$ at its peak) and the spatial structure remains more uniform (with a clear boundary near $r=0$ separating a region of $u<0$ from a region of $u>0$ ), new temporal variability appears. In particular, the flow varies at faster timescales than observed at any lower current density, as evident from the small vertical extent of colored bands in Fig. 7(a), especially near $r=-20$. Figures 4-6 vary much more slowly in time. We hypothesize that the fast oscillations at $J=350 \mathrm{~mA} / \mathrm{cm}^{2}$ are due to wavelike oscillatory motion of the Bénard cells about their equilibrium position. Wavelike motions are a common instability in convection, and such motion also explains the irregular wandering of the boundary between $u<0$ and $u>0$.

As the current density $J$ changes, the convective flow changes as well, as indicated by its speed, shape, and variability in space and time. To quantify those changes and to extract an industrially-applicable measure of mixing efficiency, we consider the mixing time (or eddy turn-over time) $\tau$,

$$
\tau=\frac{L}{\left\langle u^{2}\right\rangle^{1 / 2}},
$$

which is an order-of-magnitude estimate of the time required for one full circulation. Small mixing times correspond to more efficient mixing. We plot $\tau$ in Fig. 8 as a function of $J$, using about 


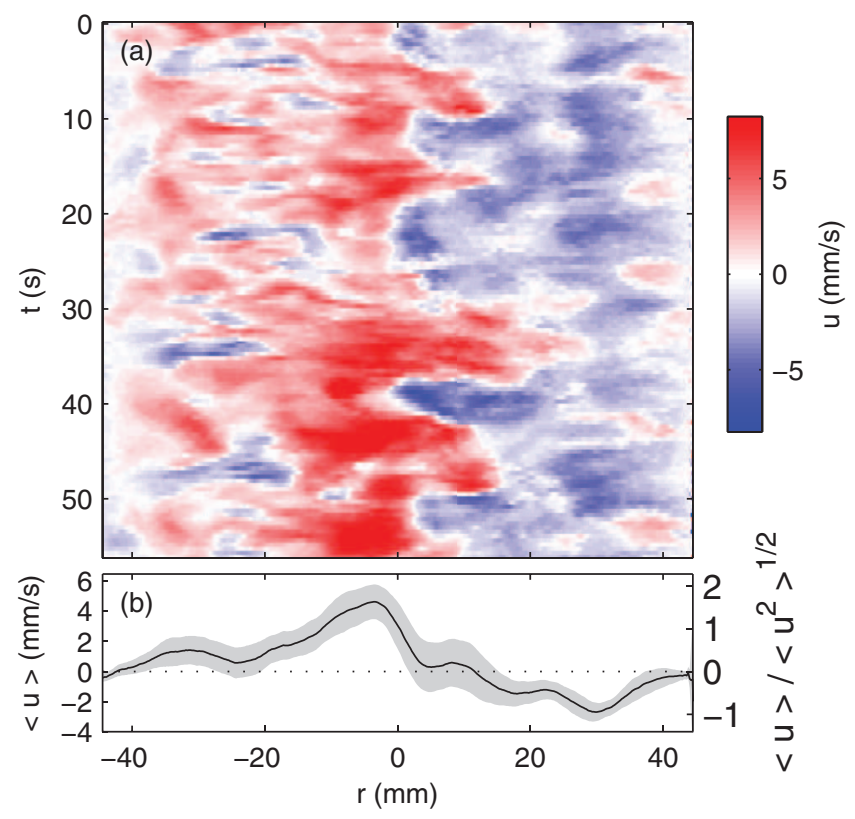

FIG. 7. Ultrasound velocity measurements of a liquid metal electrode driven by thermal convection and electrical current density $J=350 \mathrm{~mA} / \mathrm{cm}^{2}(H a=1.4)$. (a) Radial speed of thermal convection in $\mathrm{ePBBi}$, varying in both space and time, with speed indicated in color. (b) Mean radial speed, with gray region spanning one standard deviation. At $350 \mathrm{~mA} / \mathrm{cm}^{2}$, the flow shows spatial order and oscillations in time that are faster than those observed without current.

$300 \mathrm{~s}$ of velocity measurements for each data point shown. Clearly, $\tau$ tends to decrease as $J$ increases. In particular, we observe a sharp drop near $J=J_{c}=50 \mathrm{~mA} / \mathrm{cm}^{2}$. Thus we conclude that the critical current for aligning Bénard cells with magnetic field lines lies near $J_{c}$. We also attribute the slight increase in mixing time over the range $275 \mathrm{~mA} / \mathrm{cm}^{2} \leq J \leq 325 \mathrm{~mA} / \mathrm{cm}^{2}$ to the onset of wavelike motions, a second instability. Ohmic heating may also contribute to these qualitative changes in the flow. Larger current density produces more ohmic heat, which adds buoyant forces. Nor are these buoyant forces uniform; ohmic heating is most pronounced near the negative electrode, where current concentrates.

$\mathrm{Ha}$

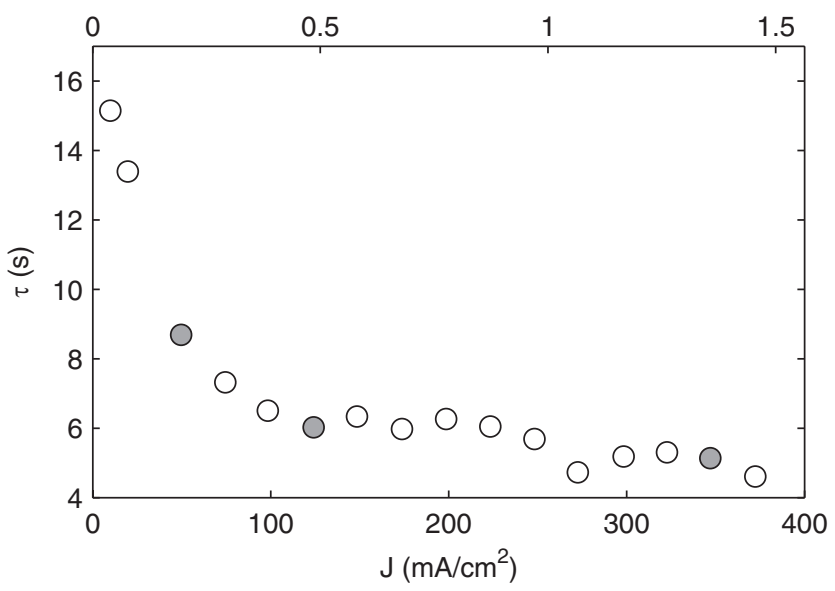

FIG. 8. Mixing time $\tau$ varying with current density $J$ and Hartmann number Ha. Flows shown in Figs. 5-7 are indicated with filled symbols. Larger currents induce faster flow and improved mixing, with a sharp transition near $J_{c}=50 \mathrm{~mA}$. 


\section{DISCUSSION AND CONCLUSIONS}

The fact that the electrode mixing time decreases as current density increases allows liquid metal batteries to operate at greater rates than would otherwise be possible. Mass transport is the likely rate-limiting step in liquid metal batteries, but the alignment of Bénard cells with magnetic field lines alleviates the limitation. In fact, extrapolating rate capability based on low-current-density measurements would substantially underestimate the true situation. Moreover, the sharp onset of mixing near $J_{c}$ is a strong motivation to maintain current density $J>J_{c}$ during much or all of the time the battery operates. By doing so, we could reduce or eliminate undesirable chemical phases. Fluid dynamical processes cannot, however, eliminate ohmic losses, which necessarily reduce energy efficiency at high charge and discharge rates.

In conclusion, we have considered mixing due to mass transport in the convecting molten electrode of a liquid metal battery. From the equation of motion we have estimated both the buoyant acceleration $\boldsymbol{a}_{b}$ and the Lorentz acceleration $\boldsymbol{a}_{L}$ to have magnitude on the order of $1 \mathrm{~mm} / \mathrm{s}^{2}$. We have used ultrasound velocimetry to measure the flow in a liquid metal electrode while varying the current density $0 \leq J \leq 375 \mathrm{~mA} / \mathrm{cm}^{2}$. We find that as the current density increases, the flow becomes faster and more regular, a fact explained by the alignment of Bénard cells with magnetic field lines. As the current density increases further, the flow develops high-frequency temporal oscillations likely due to a wavelike instability. By characterizing mixing as a function of current density using the mixing time $\tau$, we found a sharp onset in mixing efficiency at $J=J_{c}=50 \mathrm{~mA} / \mathrm{cm}^{2}$. The fact that mixing improves with current density extends the rate capability of liquid metal batteries. The fact that reversing the current direction does not reverse the flow direction allows mixing with alternating currents that do not appreciably change the state of charge of the battery.

\section{ACKNOWLEDGMENTS}

We are grateful to M. Zahn, K. Dozier, and S. Khushrushahi for generously sharing their ultrasound instrument, and for many helpful discussions. We are also grateful for the electrochemical expertise of P. Burke, B. Chung, J. M. Newhouse, T. Ouchi, H. Kim, H. Yin, X. Ning, S. Phadke, and S. A. Barriga, and B. Spatocco. This work was supported by TOTAL, SA, and the U.S. Department of Energy, Advanced Research Projects Agency-Energy (Award No. DE-AR0000047).

${ }^{1}$ M. Lei, L. Shiyan, J. Chuanwen, L. Hongling, and Z. Yan, "A review on the forecasting of wind speed and generated power,” Renew. Sust. Energ. Rev. 13, 915-920 (2009).

${ }^{2}$ C. Garrett and P. Cummins, "The power potential of tidal currents in channels," P. Roy. Soc. A-Math. Phy. 461, 2563-2572 (2005).

${ }^{3}$ S. P. Beeby, M. J. Tudor, and N. M. White, "Energy harvesting vibration sources for microsystems applications," Meas. Sci. Technol. 17, R175 (2006).

${ }^{4}$ D. H. Jeon, S. Greenway, S. Shimpalee, and J. W. Van Zee, "The effect of serpentine flow-field designs on pem fuel cell performance," Int. J. Hydrogen Energ. 33, 1052-1066 (2008).

${ }^{5}$ R. O'Hayre, S. W. Cha, W. Colella, and F. B. Prinz, Fuel Cell Fundamentals (Wiley, New York, 2009).

${ }^{6}$ R. M. Darling and P. Badrinarayanan, "Oxygen transport in polymer-electrolyte fuel cells with interdigitated air channels in porous bipolar plates," J. Electrochem. Soc. 158, B54-B60 (2011).

${ }^{7}$ A. Z. Weber, M. M. Mench, J. P. Meyers, P. N. Ross, J. T. Gostick, and Q. Liu, “Redox flow batteries: A review,” J. Appl. Electrochem. 41, 1137-1164 (2011).

${ }^{8}$ D. S. Aaron, Q. Liu, Z. Tang, G. M. Grim, A. B. Papandrew, A. Turhan, T. A. Zawodzinski, and M. M. Mench, "Dramatic performance gains in vanadium redox flow batteries through modified cell architecture," J. Power Sources 206, 450-453 (2012).

${ }^{9}$ D. J. Bradwell, H. Kim, A. H. C. Sirk, and D. R. Sadoway, "Magnesium-antimony liquid metal battery for stationary energy storage," J. Am. Chem. Soc. 134, 1895-1897 (2012).

${ }^{10}$ H. Kim, D. A. Boysen, J. M. Newhouse, B. L. Spatocco, B. Chung, P. J. Burke, D. J. Bradwell, K. Jiang, A. A. Tomaszowska, K. Wang, W. Wei, L. A. Ortiz, S. A. Barriga, S. M. Poizeau, and D. R. Sadoway, "Liquid metal batteries: Past, present, and future," Chem. Rev. 113, 2075-2099 (2013).

${ }^{11}$ Liquid metal batteries are being commercialized by Ambri Inc., Cambridge, MA.

${ }^{12}$ J. M. Aurnou and P. L. Olson, "Experiments on Rayleigh-Bénard convection, magnetoconvection and rotating magnetoconvection in liquid gallium," J. Fluid Mech. 430, 283-307 (2001).

${ }^{13}$ E. M. King and J. M. Aurnou, "Turbulent convection in liquid metal with and without rotation," Proc. Natl. Acad. Sci. U.S.A. 110, 6688-6693 (2013).

${ }^{14}$ S. Horanyi, L. Krebs, and U. Müller, “Turbulent Rayleigh-Bénard convection in low Prandtl-number fluids," Intl. J. Heat Mass Transf. 42, 3983-4003 (1999). 
${ }^{15}$ R. Verzicco and R. Camussi, "Prandtl number effects in convective turbulence," J. Fluid Mech. 383, 55-73 (1999).

${ }^{16}$ S. Grossmann and D. Lohse, "Scaling in thermal convection: A unifying theory," J. Fluid Mech. 407, 27-56 (2000).

${ }^{17}$ H. T. Rossby, "A study of Bénard convection with and without rotation," J. Fluid Mech. 36, 309-335 (1969).

${ }^{18}$ V. Bojarevičs, Ya. Freibergs, E. I. Shilova, and E. V. Shcherbinin, Electrically Induced Vortical Flows (Kluwer Academic Publishers, Dordrecht, 1989).

${ }^{19}$ H. K. Moffatt, "Electromagnetic stirring," Phys. Fluids A 3, 1336-1343 (1991).

${ }^{20}$ A. Cramer, G. Gerbeth, P. Terhoeven, and A. Krätzschmar, "Fluid velocity measurements in electro-vortical flows," Mater. Manufact. Proces. 19, 665-678 (2004).

${ }^{21}$ O. Kazak and O. Semko, "Modelling vortex fields in metal smelting furnaces," Int. J. Multiphys. 4, 351-358 (2010).

${ }^{22}$ D. Brito, H-C. Nataf, P. Cardin, J. Aubert, and J-P. Masson, "Ultrasonic doppler velocimetry in liquid gallium," Exp. Fluids 31, 653-663 (2001).

${ }^{23}$ S. Eckert and G. Gerbeth, "Velocity measurements in liquid sodium by means of ultrasound Doppler velocimetry," Exp. Fluids 32, 542-546 (2002).

${ }^{24}$ Y. Takeda and H. Kikura, "Flow mapping of the mercury flow," Exp. Fluids 32, 161-169 (2002).

${ }^{25}$ B. E. Brawn, K. Joshi, D. P. Lathrop, N. Mujica, and D. R. Sisan, "Visualizing the invisible: Ultrasound velocimetry in liquid sodium," Chaos 15, 041104 (2005).

${ }^{26}$ S. Eckert, A. Cramer, and G. Gerbeth, "Velocity measurement techniques for liquid metal flows," Magnetohydrodynamics, Fluid Mechanics and Its Applications, Vol. 80 (Springer Netherlands, 2007), pp. 275-294.

${ }^{27}$ Y. Yao, M. T. McDowell, I. Ryu, H. Wu, N. Liu, L. Hu, W. D. Nix, and Y. Cui, "Interconnected silicon hollow nanospheres for lithium-ion battery anodes with long cycle life," Nano Lett. 11, 2949-2954 (2011).

${ }^{28}$ R. A. Huggins, "Lithium alloy negative electrodes," J. Power Sour. 81-82, 13-19 (1999).

${ }^{29}$ R. J. Tayler, "Hydromagnetic instabilities of an ideally conducting fluid," Proc. Phys. Soc. Lond. B 70, 31 (1957).

${ }^{30}$ R. J. Tayler, "The adiabatic stability of stars containing magnetic fields. I. Toroidal fields," Mon. Not. R. Astron. Soc. 161, 365 (1973), available at http://adsabs.harvard.edu/

${ }^{31}$ G. Rüdiger and M. Schultz, "Tayler instability of toroidal magnetic fields in MHD Taylor-Couette flows," Astron. Nachr. 331, 121-129 (2010).

${ }^{32}$ M. Seilmayer, F. Stefani, T. Gundrum, T. Weier, G. Gerbeth, M. Gellert, and G. Rüdiger, "Experimental evidence for a transient Tayler instability in a cylindrical liquid-metal column,” Phys. Rev. Lett. 108, 244501 (2012).

${ }^{33}$ F. Stefani, T. Weier, T. Gundrum, and G. Gerbeth, "How to circumvent the size limitation of liquid metal batteries due to the Tayler instability," Energ. Convers. Manage. 52, 2982-2986 (2011).

${ }^{34}$ J. M. Newhouse, "Modeling the operating voltage of liquid metal battery cells," Ph.D. thesis (Massachusetts Institute of Technology, 2014).

${ }^{35}$ H. Kim, D. A. Boysen, D. J. Bradwell, B. Chung, K. Jiang, A. A. Tomaszowska, K. Wang, W. Wei, and D. R. Sadoway, "Thermodynamic properties of calcium-bismuth alloys determined by emf measurements," Electrochim. Acta 60, 154-162 (2012).

${ }^{36}$ S. Poizeau, H. Kim, J. M. Newhouse, B. L. Spatocco, and D. R. Sadoway, "Determination and modeling of the thermodynamic properties of liquid calcium-antimony alloys,” Electrochim. Acta 76, 8-15 (2012).

${ }^{37}$ J. M. Newhouse, S. Poizeau, H. Kim, B. Spatocco, and D. R. Sadoway, "Thermodynamic properties of calcium-magnesium alloys determined by emf measurements," Electrochim. Acta 91, 293-301 (2013).

${ }^{38}$ H. Kim, D. A. Boysen, T. Ouchi, and D. R. Sadoway, "Calcium-bismuth electrodes for large-scale energy storage (liquid metal batteries)," J. Power Sour. 241, 239-248 (2013).

${ }^{39}$ Handbook on Lead-Bismuth Eutectic Alloy and Lead Properties, Materials Compatibility, Thermal-Hydraulics, and Technologies (Nuclear Energy Agency, 2007).

${ }^{40}$ G. Ahlers, "Turbulent convection," Physics 2, 74 (2009).

${ }^{41}$ S. Eckert, G. Gerbeth, and V. I. Melnikov, "Velocity measurements at high temperatures by ultrasound doppler velocimetry using an acoustic wave guide," Exp. Fluids 35, 381-388 (2003).

${ }^{42}$ B. Lehnert and N. C. Little, "Experiments on the effect of inhomogeneity and obliquity of a magnetic field in inhibiting convection," Tellus 9, 97-103 (1957).

${ }^{43}$ S. Fauve, C. Laroche, and A. Libchaber, "Effect of a horizontal magnetic field on convective instabilities in mercury," J. Phys. Lett. 42, 455-457 (1981).

${ }^{44}$ S. Cioni, S. Ciliberto, and J. Sommeria, "Strongly turbulent Rayleigh-Bénard convection in mercury: Comparison with results at moderate Prandtl number," J. Fluid Mech. 335, 111-140 (1997).

${ }^{45} \mathrm{U}$. Burr and U. Müller, "Rayleigh-Bénard convection in liquid metal layers under the influence of a horizontal magnetic field," J. Fluid Mech. 453, 345-369 (2002).

${ }^{46}$ E. Brown, A. Nikolaenko, and G. Ahlers, "Reorientation of the large-scale circulation in turbulent rayleigh-bénard convection," Phys. Rev. Lett. 95, 084503 (2005).

${ }^{47}$ Y. Tsuji, T. Mizuno, T. Mashiko, and M. Sano, "Mean wind in convective turbulence of mercury," Phys. Rev. Lett. 94, 034501 (2005).

${ }^{48}$ E. Brown and G. Ahlers, "Large-scale circulation model for turbulent Rayleigh-Bénard convection," Phys. Rev. Lett. 98, 134501 (2007). 